\title{
Diffusion Monte Carlo Calculations for Rare-earths: Hartree-Fock, Hybrid B3LYP, and Long-range Corrected LC-BLYP Functional
}

\author{
Nagat Elkahwagy $^{1, *}$, Atif Ismail ${ }^{1,2}$, S. M. A. Maize ${ }^{3}$, K. R. Mahmoud ${ }^{1}$ \\ ${ }^{1}$ Department of Physics, Faculty of Science, Kafrelsheikh University, Egypt \\ ${ }^{2}$ Department of Physics, Faculty of Applied Sciences, Umm Al Qura University, Saudi Arabia \\ ${ }^{3}$ Department of Physics, Faculty of Science, Menoufia University, Egypt
}

Copyright $\subseteq 2016$ by authors, all rights reserved. Authors agree that this article remains permanently open access under the terms of the Creative Commons Attribution License 4.0 International License

\begin{abstract}
Our study aim is to make highlights on the failure or success of both popular B3LYP functional and long-range corrected LC-BLYP functional at different values of the range separation parameter in improving the ground state and excited states energies calculations for 4f-lanthanides and $5 \mathrm{f}$-actinides. The most popular B3LYP functional fails to provide a balanced description of excitation energies for $4 \mathrm{f}$-lanthanides. However, it gives reasonable results for the actinides with exception of $\mathrm{Pu}$ and $\mathrm{Am}$ atoms. The ground state energies of $5 \mathrm{f}$-actinides are improved significantly at the critical value of the range separation parameter equals 0.35 a.u. while the optimal value for the excited states lies in the critical range of 0.35-0.4 a.u. However, our results show that LC-BLYP functional is not recommended for 4f-lanthanides which have strongly localized nature.
\end{abstract}

Keywords Diffusion Monte Carlo, Density Functional Theory, Rare-earths

\section{Introduction}

Obtaining accurate results for rare-earths is still a challenging problem. The difficulties presented by these elements in quantum mechanical calculations arise from the large magnitude of the relativistic effect and the limitation in the electron correlation treatment. Quantum Monte Carlo (QMC) methods [1-4] are among the most accurate to predict relatively accurate properties of quantum systems. Besides its favorable scaling with system size, any arbitrarily complex wave function can be used because the integrals are evaluated numerically. Although there are many different QMC approaches, diffusion Monte Carlo (DMC) remains the most accurate one.

In recent years, there has been a concerned effort to improve the quality of QMC results in order to arrive to chemical accuracy. In fact, many papers [5-7] have employed the density functional theory (DFT) particularly the most successful functional Becke 3 parameter- LeeYang- Parr (B3LYP) as a starting point to produce orbitals to quantum Monte Carlo calculations and highly accurate results have been obtained.

Nevertheless, the standard B3LYP functional contains only short-range HF exchange so the long-range asymptotic behavior still remains incorrect. The introduction of long-range HF exchange in DFT functional will correct the description of long-range exchange interaction. So the long-range corrected functionals are providing a considerable improvement with respect to the hybrid functionals. The general form of the long-range corrected functional is

$$
\begin{aligned}
& E_{x c}{ }^{L C-D F T}=E_{c}{ }^{D F T}+\left(1-C_{H F}\right) E_{x}{ }^{S R-D F T}+ \\
& +C_{H F} E_{x}^{S R-H F}+E_{x}{ }^{L R-H F}
\end{aligned}
$$

where the traditional exchange-correlation functional $E_{x c}$ may be a pure or hybrid functional. The components labeled "LR" and "SR" are evaluated using the long-range and short-range Coulomb potentials, respectively, while $C_{H F}$ denotes the amount of HF exchange present in the original functional.

In this paper, we perform DMC calculations of the ground and excited states energies for some rare-earths by using both HF and DFT (B3LYP) orbitals to investigate the performance of each for f-elements. Indeed, we study the dependence of the long-range corrected LC-BLYP functional on the range separation parameter $\mu$ and examine its performance for rare-earths. The basic form of the wave function that we used is the Slater-Jastrow wave function which is considered the most common and simplest one. In the next section, we outline a brief description of the DMC method. The results are then presented and discussed. 
Finally, we give the conclusion of this work.

\section{Computational Methods}

Diffusion Monte Carlo method has been extensively described in the literatures [8-10] so we give here a brief description of it. The diffusion Monte Carlo (DMC) method is a stochastic projector method for solving the imaginary time many-body Schrödinger equation:

$$
-\frac{\partial}{\partial \tau} \psi(R, \tau)=\left(-\frac{1}{2} \nabla^{2}+V-E_{T}\right) \psi(R, \tau)
$$

where $\tau$ is the imaginary time, $\tau=i t$ and $E_{T}$ is the energy offset.

Importance sampling with a trial wave function $\psi_{T}(R)$ is used to improve the statistical accuracy of the simulation and this is can be achieved by multiplying Eq. (2) by $\psi_{T}(R)$ and rearranging

$$
\begin{aligned}
& -\frac{\partial f(R, \tau)}{\partial \tau}=-\frac{1}{2} \nabla^{2} f(R, \tau)+ \\
& +\nabla \cdot\left[f(R, \tau) \cdot v_{D}(R)\right]+\left[E_{L}-E_{T}\right] f(R, \tau)
\end{aligned}
$$

where $f(R, \tau)=\psi(R, \tau) \psi_{T}(R) \quad$ interpreted $\quad$ as $\quad$ a probability density and $E_{L}(R) \frac{\hat{H} \psi_{T}(R)}{\psi_{T}(R)}$ is the local energy. this equation can be simulated with a random walk having diffusion, a draft, and a branching step and may be written in the integral form:

$$
f(R, \tau+\Delta \tau)=\int G\left(R, R^{\prime} ; \Delta \tau\right) \cdot f(R, \tau) d R
$$

Where the Green's function $G\left(R, R^{\prime} ; \Delta \tau\right)$ is a solution of the same equation (3) initial and can be interpreted as a probability of transition from a state $\mathrm{R}$ to $\mathrm{R}$ '. It is possible to use MC method to solve the integral in Eq. (4) but the difficulty is that the precise form of $G\left(R, R^{\prime} ; \Delta \tau\right)$ is not known. Fortunately the comparison of the Schrödinger equation with the diffusion equation gives us a clue about how one might approximate the unknown Green's function.

The evolution during the long time interval $\tau$ can be generated repeating a large number of short time steps $\tau$. In the limit $\tau \rightarrow 0$, one can make use of the short time approximation for Green's function [11]:

$$
\begin{gathered}
G\left(R, R^{\prime} ; \Delta \tau\right) \approx(2 \pi \Delta \tau)^{-3 N / 2} \exp \left[-\frac{\left(R-R^{\prime}-\Delta \tau \nabla \ln \left|\psi_{T}\right|^{2}\right)^{2}}{2 \Delta \tau}\right] \\
. \exp \left[-\Delta \tau\left[E_{L}\left(R^{\prime}\right)+E_{L}(R)-2 E_{T}\right] / 2\right]
\end{gathered}
$$

But due to the fermionic nature of electrons, the wave function must have positive and negative parts and this is opposite with the assumed nature of $\psi$ which is a probability distribution. So the fixed-node approximation [12] had been used to deal with the fermionic antisymmetry which constrains the nodal surface of $\psi$ to equal that of the antisymmetric trial wave function $\psi_{T}$.

In our calculations, the wave function was generated by using the quantum chemistry program GAMESS [13]. The Slater determinant was obtained from HF and DFT (B3LYP) methods in order to check the accuracy of each on this type of systems. We make use of CRENBL ECP [14] basis set for all elements except La atom where CRENBS ECP basis set is being used which proved to be successful for the calculations. The program package Qwalk [15] code is used for the QMC calculations. The present DMC calculations are pseudopotential calculations done with a very small time step, $\tau=0.001 H^{-1}$, to remove the time step error within statistical uncertainties. We also use a mean population of 2000 walkers.

\section{Results and Discussion}

We summarize our results for the ground and excited states for some rare earths using both HF and B3LYP orbitals in tables 1 and 2. The electronic excitation involves a promotion of an electron with a change of spin from $6 \mathrm{~s} \rightarrow 5 \mathrm{~d}$ and $7 \mathrm{~s} \rightarrow 6 \mathrm{~d}$ for lanthanides and actinides respectively. The excitation energies have been calculated directly from the difference between the excited and the ground state energy. In table 3 we list the calculated excitation energies along with the experimental values [16] for the sake of comparison. Unfortunately, no experimental data for Pm and Pu atoms are available.

It is clearly seen from the tables 1 and 2 that B3LYP performs better than HF for La, Ac, and Th, elements with no f-electrons. However, the situation is different when $4 \mathrm{f}$ and $5 \mathrm{f}$ subshells are being populated in lanthanides and actinides respectively.

At first our results show that for lanthanides having electrons in their $4 \mathrm{f}$ subshell, B3LYP performs worse than HF. The standard B3LYP functional produces unphysical results; the excited state energy is lower than the energy of ground state for many atoms. Indeed, self-consistent field (SCF) fails to converge on the first step in Gamess. As indicated in table 3 the calculated excitation energies employing B3LYP functional yield negative excitation energies for all $4 \mathrm{f}$-lanthanides except for $\mathrm{Ce}$ and $\mathrm{Pm}$ atoms. These results confirm that the most popular B3LYP fails to calculate the excitation energy for 4f-lanthanides. In Fact, we believe that the poor performance of B3LYP functional in $4 \mathrm{f}$-lanthanides is traced back to the strong localization of $4 \mathrm{f}$-electrons. This failure is due to in part to the so called "self-interaction error" which means incomplete cancellation between the self- Coulomb term and approximate self-exchange contribution. On the other hand, the hybrid 
B3LYP seems to be quite satisfactory for actinides especially in the case of $\mathrm{Pa}$ atom. However, for both $\mathrm{Pu}$ and $\mathrm{Am}$ atoms negative excitation energies have been obtained. The failure of standard B3LYP for Am atom is attributed to the well-known fact that starting with Am atom to the end of actinide series; the 5f-states are localized and resemble the $4 \mathrm{f}$-states in lanthanides. So we predict that B3LYP functional is unable to deal with actinides at the end of the series.

Table 1. Ground state energies $G$ and excited state energies $E$ (due to 6s-5d transition) computed within DMC for lanthanides using both $\mathrm{HF}$ and B3LYP orbitals. All energies are in Hartrees.

\begin{tabular}{|c|c|c|c|}
\hline Atom & State & HF & B3LYP \\
\hline La & G & -1.2587 & -1.2556 \\
& E & -1.2089 & -1.2407 \\
& G & -2.7421 & -2.7138 \\
Pr & E & -2.6646 & -2.5894 \\
& G & -5.5754 & -4.7310 \\
Nd & E & -5.5290 & -5.3237 \\
& G & -9.3234 & -9.3288 \\
Pm & E & -9.1617 & -9.3609 \\
& G & -14.2717 & -14.3620 \\
Sm & G & -20.4260 & -14.0353 \\
& E & -20.5096 & -20.2920 \\
Eu & G & -28.6600 & -28.6630 \\
& E & -28.6508 & -28.7529 \\
\hline
\end{tabular}

Table 2. Ground state energies $G$ and excited state energies $E$ (due to $7 \mathrm{~s}-6 \mathrm{~d}$ transition) computed within DMC for actinides using both $\mathrm{HF}$ and B3LYP orbitals. All energies are in Hartrees.

\begin{tabular}{|c|c|c|c|}
\hline Atom & State & HF & B3LYP \\
\hline Ac & G & -29.4745 & -29.4865 \\
& E & -29.4010 & -29.4374 \\
& G & -35.6613 & -35.6253 \\
$\mathrm{~Pa}$ & E & -35.5690 & -35.5777 \\
& G & -42.6303 & -42.6491 \\
$\mathrm{U}$ & E & -42.6083 & -42.5859 \\
& G & -51.2107 & -51.2060 \\
$\mathrm{~Np}$ & E & -51.1881 & -51.1979 \\
& G & -60.4527 & -60.5301 \\
$\mathrm{Pu}$ & E & -60.2922 & -60.4908 \\
& G & -71.1153 & -71.3357 \\
$\mathrm{Am}$ & $\mathrm{E}$ & -70.9784 & -71.3974 \\
& $\mathrm{G}$ & -74.6698 & -74.5900 \\
& $\mathrm{E}$ & -74.7166 & -74.8191 \\
\hline
\end{tabular}

Table 3. Excited state energy values for rare earths $(\mathrm{eV})$. Values were calculated using HF and B3LYP orbitals.

\begin{tabular}{|c|c|c|c|}
\hline Atom & HF & B3LYP & EXP \\
\hline $\mathrm{La}$ & 1.35 & 0.40 & 0.33 \\
$\mathrm{Ce}$ & 2.10 & 3.38 & 1.06 \\
$\mathrm{Pr}$ & 1.26 & -20.30 & 1.00 \\
$\mathrm{Nd}$ & 4.23 & -0.87 & 1.05 \\
$\mathrm{Pm}$ & 1.71 & 8.88 & - \\
$\mathrm{Sm}$ & -2.27 & -16.00 & 1.33 \\
$\mathrm{Eu}$ & 0.25 & -2.44 & 1.60 \\
$\mathrm{Ac}$ & 1.99 & 1.33 & 1.14 \\
$\mathrm{Th}$ & 2.48 & 1.29 & 0.69 \\
$\mathrm{~Pa}$ & 1.35 & 1.14 & 0.87 \\
$\mathrm{U}$ & 0.61 & 0.22 & 0.77 \\
$\mathrm{~Np}$ & 4.36 & 1.06 & 0.88 \\
$\mathrm{Pu}$ & 3.72 & -1.67 & - \\
$\mathrm{Am}$ & -1.27 & -6.23 & 1.79 \\
\hline
\end{tabular}

Otherwise, it is well known that in the hybrid DFT functional a part of the short-range HF exchange is mixed into the semi-local DFT exchange. The hybrid B3LYP functional contains $20 \%$ short range $\mathrm{HF}$ exchange. As has been described previously, the hybrid functional with only short-range $\mathrm{HF}$ exchange is insufficient to describe the ground and excited states of f-elements. In an attempt to study the influence of inclusion of long-range HF exchange on rare earths, we calculate the ground and the excited states energies by using the long-range corrected LC-BLYP functional (which combine long-range HF exchange with short-range BLYP exchange and using a standard full-range DFT correlation). To this aim, we study the performance of the latter functional at different values of the range separation parameter $\mu$ in comparison to pure BLYP functional for both lanthanides and actinides.

Table 4. Ground state energies $G$ and excited state energies $E$ (due to $6 \mathrm{~s}-5 \mathrm{~d}$ transition) computed within DMC for lanthanides using LC-BLYP functional as a function of $\mu$ compared to pure BLYP functional

\begin{tabular}{|c|c|c|c|c|c|}
\hline Method & State & La & Ce & Pr & Eu \\
\hline BLYP & G & -1.2558 & -2.8592 & -4.7222 & -26.9003 \\
& E & -1.2392 & -2.5494 & -5.4071 & -27.5902 \\
\hline LC-BLYP & G & \multicolumn{5}{|l|}{} \\
\hline$\mu=0.30$ & G & -1.2537 & -2.9310 & -5.5725 & -28.6687 \\
& E & -1.2412 & -2.7996 & -5.5303 & -28.6498 \\
$\mu=0.35$ & G & -1.2568 & -2.8961 & -5.6074 & -28.5934 \\
& E & -1.2412 & -2.6643 & -5.5542 & -28.7263 \\
$\mu=0.40$ & G & -1.2564 & -2.8292 & -5.5089 & -28.7909 \\
& E & -1.2412 & -2.5428 & -5.4976 & -28.8660 \\
$\mu=0.45$ & G & -1.2518 & -2.8617 & -5.5882 & -28.6728 \\
& E & -1.2068 & -2.6172 & -5.5245 & -28.6895 \\
& G & -1.2546 & -2.8772 & -5.5794 & -28.5903 \\
& E & -1.2069 & -2.7719 & -5.5299 & -28.8503 \\
\hline
\end{tabular}


Regarding lanthanides, the inclusion of long-range HF exchange at different values of $\mu$ fails to converge Gamess calculations for $\mathrm{Nd}, \mathrm{Pm}$, and $\mathrm{Sm}$ atoms. So we do not include here the results of them, however, the results of other lanthanides are summarized in table 4 . It is clearly seen that both the ground and excited states energies of Ce atom are improved considerably at $\mu=0.3$, while the optimal value of the range separation parameter for Pr element equals 0.4. It can also be observed that LC-BLYP functional performs rather well for $\mathrm{Eu}$ especially at $\mu=0.4$ whereas for $\mathrm{La}$, there is a slight improvement at $\mu=0.35$.

Note that $\mathrm{Ce}$ is the only one element exhibits $4 \mathrm{f}$ itinerant behavior so the observed improvement by using LC-BLYP is not surprising. On the other hand, despite $\mathrm{Eu}$ atom has strongly localized 4 f-electrons, the use of LC-BLYP at the optimal value of $\mu$ enhances the results considerably which is a direct consequence of its half filled electronic configuration that acquires more stability to the atom.

It is also interesting to point out that although $4 \mathrm{f}$-electrons are localized in Pr atom, a clearly improvement has been observed by applying the long range corrected scheme to BLYP functional. This behavior supports P. Lethuillier et al [17] observation who confirmed that the localization of praseodymium compounds is intermediate between those of cerium and neodymium.

The calculated results of actinides are listed in table 5. The ground state energies for $\mathrm{U}, \mathrm{Np}, \mathrm{Pu}$, and $\mathrm{Am}$ atoms as a function of the range separation parameter $\mu$ ranging from 0.3 a.u. to 0.5 a.u. are indicated in Fig. 1 . It should be noted that we also investigate the performance outside the range [0.3:0.5] a.u. but no significant improvement can be achieved.

Our calculations found that the addition of long-range HF exchange to pure BLYP functional does not alter the results significantly for Ac and Th atoms, actinide elements with no 5f-electrons, compared to the pure BLYP functional. In contrast, the presence of the long-range HF exchange clearly enhances the ground state energy for 5 f-actinides, except $\mathrm{Pa}$ atom, at the value of $0.35 \mathrm{a}$. u. of the range separation parameter as indicated in Fig. 1. This value gives the lowest DMC ground state energy for all atoms indicated in the figure with the exception of the $\mathrm{Pu}$ atom where a comparable energy with the energy of $\mu=0.45$ a.u. has been obtained. Like $\mathrm{Eu}$, the improvement gained for Am atom is related to its half filled electronic configuration. On the other hand, the excited state energies have been improved considerably at the value around the range 0.35 a.u. -0.4 a.u. which is slightly higher than the optimal value for the ground state.

Table 5. Ground state energies $G$ and excited state energies $E$ (due to 7s-6d transition) computed within DMC for actinides using LC-BLYP functional as a function of $\mu$ compared to pure BLYP functional.

\begin{tabular}{|c|c|c|c|c|c|c|c|c|}
\hline Method & State & Ac & Th & $\mathbf{P a}$ & $\mathbf{U}$ & $\mathbf{N p}$ & $\mathbf{P u}$ & Am \\
\hline \multirow[t]{2}{*}{ BLYP } & G & -29.4850 & -35.6742 & -42.6430 & -51.2933 & -60.5910 & -51.8073 & -74.5963 \\
\hline & E & -29.4421 & -35.5662 & -42.5987 & -51.1612 & -60.4432 & -52.8309 & -74.5432 \\
\hline \multicolumn{9}{|l|}{ LC-BLYP } \\
\hline \multirow[t]{2}{*}{$\mu=0.30$} & G & -29.4730 & -35.6471 & -42.6482 & -51.2226 & -60.4301 & -71.3359 & -74.6117 \\
\hline & E & -29.4144 & -35.5520 & -42.6211 & -51.1889 & -604509 & -71.2793 & -74.5618 \\
\hline \multirow[t]{2}{*}{$\mu=0.35$} & G & -29.4749 & -35.6445 & -42.6880 & -51.3280 & -60.6486 & -71.4135 & -74.7796 \\
\hline & E & -29.3970 & -35.5769 & -41.9985 & -51.2047 & -60.6103 & -71.2421 & -74.8217 \\
\hline \multirow[t]{2}{*}{$\mu=0.40$} & G & -29.4787 & -35.6510 & -42.6774 & -51.2510 & -60.5861 & -71.4043 & -74.6294 \\
\hline & E & -29.3778 & -35.5817 & -42.7063 & -51.1696 & -60.3880 & -71.2819 & -74.3282 \\
\hline \multirow[t]{2}{*}{$\mu=0.45$} & G & -29.4551 & -35.6289 & -42.7299 & -51.2401 & -60.5416 & -71.4243 & -74.4759 \\
\hline & $\mathrm{E}$ & -29.3987 & -35.6089 & -42.5798 & -51.1591 & -60.4513 & -71.2508 & -74.6854 \\
\hline \multirow[t]{2}{*}{$\mu=0.50$} & G & -29.4666 & -35.6446 & -42.6883 & -51.1346 & -60.6306 & -71.3770 & -74.6021 \\
\hline & E & -29.3771 & -35.6081 & -42.6553 & -51.1705 & -60.4331 & -71.2181 & -74.5084 \\
\hline
\end{tabular}



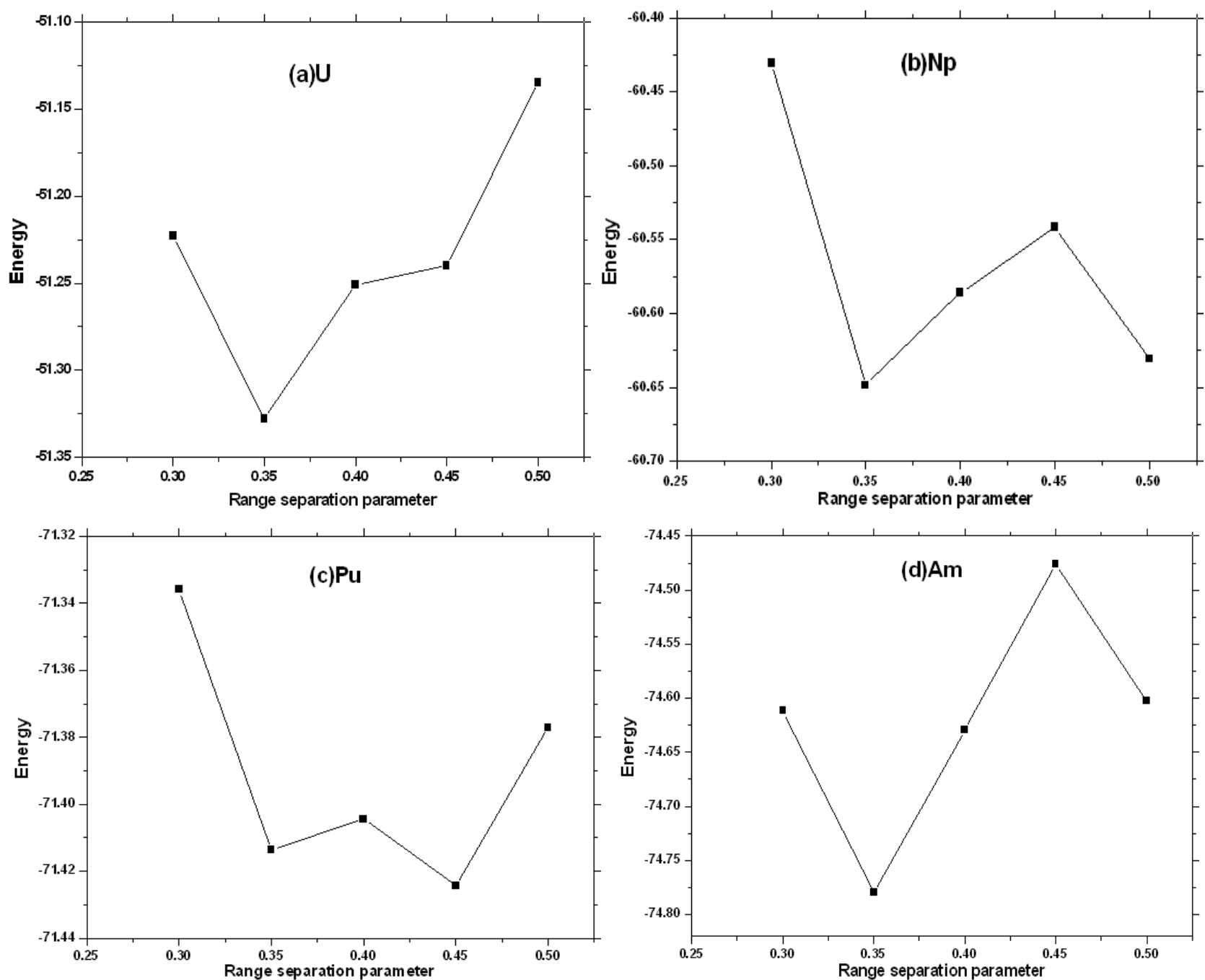

Figure 1. Ground state energy for some actinides as a function of the range separation parameter.

Our recommended value for the range separated parameter whatsoever either in the ground or excited state is close to the value of $\mu=0.4$ a.u. suggested by Gerber et al. [18] for molecular systems. Otherwise, to some extent our value is comparable to the optimal value $\mu=0.45$ a.u. presented by Rohrdanz et al. [19] and Henderson et al. [20] for the ground state thermodynamic.

Finally, the results in this work support that LC-BLYP functional provides good results for 5 f-actinides and some lanthanides having less localized character of $4 \mathrm{f}$-electrons, however, this paper points out the shortcoming of the latter functional to deal with strongly localized systems. In fact, the inclusion of the long-range HF exchange corrects the asymptotic behavior of the exchange potential and removes the long-range self-interaction error which the conventional hybrid functionals is incapable to remove it. But from our point of view, the failure is mostly related to the LYP correlation which is not suited for strongly localized systems. So we intend to investigate the performance of applying the long-range corrected scheme to other exchange-correlation functionals not having the common LYP correlation in a future study.

\section{Conclusions}

By using the diffusion Monte Carlo (DMC) method, we investigate the performance of both HF and B3LYP orbitals for some rare earths. It was found that B3LYP functional works worse than Hartree-Fock for all 4f-lanthanides. Whereas, the same functional gives acceptable results for actinides with exception of $\mathrm{Pu}$ and Am atoms. We further test the performance of the long-range corrected LC-BLYP functional at different values of the range separation parameter $\mu$. Unfortunately, the latter functional does not improve the calculations for most 4f-lanthanides which strongly localized character. Conversely, it enhances both the ground state energies of $5 \mathrm{f}$-actinides at the value $\mu=0.35$ a.u. and the excited state energies around the range of 0.35-0.4 a.u.

\section{Acknowledgements}

The authors gratefully thank Dr. Lucas Wagner for helpful discussions in Qwalk quantum Monte Carlo software. 


\section{REFERENCES}

[1] J. B. Anderson. Quantum Monte Carlo. Origins, Development, Applications, Oxford University press, 2007.

[2] M. P. Nightingale and C. J. Umrigar. Quantum Monte Carlo Methods in Physics and Chemistry, Kluwer Academic Publishers, 1999.

[3] W. M. C. Foulkes, L. Mitas, R. J. Needs and G. Rajagopal. Quantum Monte Carlo simulations of solids, Rev. Mod. Phys. Vol. 73 33, 2001.

[4] L. M. Sobol. A primer for the Monte Carlo Method, CRC Press, 1994

[5] L. Wagner and L. Mitas. A quantum Monte Carlo study of electron correlation in transition metal oxygen molecules, Chem. Phys. Lett. Vol. 370 412, 2003.

[6] E. Sola, J. P. Brodholt, and D. Alfè. Equation of state of hexagonal closed packed iron under Earth's core conditions from quantum Monte Carlo calculations, Phys. Rev. B Vol. $79024107,2009$.

[7] Shi-Guo. Quantum Monte Carlo Studies of Ultra-Cold Molecules and Rashba Interactions, Ph. D. Thesis, University of North California State, 2014.

[8] R J Needs, M D Towler, N D Drummond and P López Ríos. Continuum variational and diffusion quantum Monte Carlo calculations, J. Phys. Cond. Matt. Vol. 22 023201, 2009.

[9] C. J. Umrigar, M. P. Nightingale, and K. J. Runge. A diffusion Monte Carlo algorithm with very small time step errors, J. Chem. Phys. Vol. 99 2865, 1993.

[10] D. Ceperley and B. Alder. Quantum Monte Carlo, Science
Vol. $231555,1986$.

[11] W. A. Lester, S. M. Rothstein, and S. Tanaka. Recent Advances in Quantum Monte Carlo Methods: Part II, World Scientific Publishing Co. Pte. Ltd., 2002.

[12] J. B. Anderson., Quantum chemistry by random walk. $\mathrm{H}^{2} \mathrm{P}$, $\mathrm{H}_{3}^{+} \mathrm{D}_{3 \mathrm{~h}}{ }^{1} \mathrm{~A}_{1}^{\prime}, \mathrm{H}_{2}{ }^{3} \Sigma^{+}{ }_{\mathrm{u}}, \mathrm{H}_{4}{ }^{1} \Sigma^{+}{ }_{\mathrm{g}}$, be ${ }^{1} \mathrm{SJ}$, Chem. Phys. Vol. 65 4121,1976 .

[13] M. W. Schmidt, J. A. Boatz, K. K. Baldridge, S. T. Elbert, M. S. Gordon, J. H. Jensen, S. Koseki, N. Matsunaga, K. A. Nguyen, S. Su, T. L. windus, M. Dupuis, and J. A. Montgomery. General atomic and molecular electronic structure system, J. Comp. Chem. Vol. 14 1347, 1993.

[14] D. Feller. The Role of Databases in Support of Computational Chemistry Calculations, J. Comp. Chem. Vol. 17 1571, 1996.

[15] L. K. Wagner, M. Bajdich, and L. Mitas. QWalk: A Quantum Monte Carlo Program for Electronic Structure, J. Comp. Phys. Vol. 228 3390, 2009.

[16] J. E. Sansonetti and W. C. Martin. Handbook of Basic Atomic Spectroscopic Data, NIST online database, National Institute of Standards and Technology, Gaithersburg, Maryland, 2005.

[17] P. Lethuillier, J. Chaussy. Sign change of the c.e.f. parameters in light rare earth compounds in relation with the delocalization of the 4f shell, J. Phys. Paris 37 123, 1976.

[18] I. C. Gerber and J. G. Angyan. Hybrid functional with separated range, Chem. Phys. Lett. Vol. 415 100, 2005.

[19] Mary A. Rohrdanz, Katie M. Martins and John M. Herbert. A long-range corrected density functional that performs well for both ground-state properties and time- dependent density functional theory excitation energies, including charge transfer excited states, J. Chem. Phys. Vol. 130 054112, 2009.

[20] T. N. Henderson, B. G. Janesko, and G. E. Scuseria. Generalized gradient approximation model exchange holes for range-separated hybrids, J. Chem. Phys. Vol. 128 3540, 2008. 